\title{
Ultrasonographic assessment of parasternal intercostal muscles during mechanical ventilation
}

\author{
Paolo Formenti ${ }^{*}$, Michele Umbrello ${ }^{1}$, Martin Dres ${ }^{2,3}$ and Davide Chiumello ${ }^{1,4,5}$
}

\begin{abstract}
Although mechanical ventilation is a lifesaving treatment, abundant evidence indicates that its prolonged use (1 week or more) promotes respiratory muscle weakness due to both contractile dysfunction and atrophy. Along with the diaphragm, the intercostal muscles are one of the most important groups of respiratory muscles. In recent years, muscular ultrasound has become a useful bedside tool for the clinician to identify patients with respiratory muscle dysfunction related to critical illness and/or invasive mechanical ventilation. Images obtained over the course of illness can document changes in muscle dimension and can be used to estimate changes in function. Recent evidence suggests the clinical usefulness of ultrasound imaging in the assessment of intercostal muscle function. In this narrative review, we summarize the current literature on ultrasound imaging of the parasternal intercostal muscles as used to assess the extent of muscle activation and muscle weakness and its potential impact during discontinuation of mechanical ventilation. In addition, we proposed a practical flowchart based on recent evidence and experience of our group that can be applied during the weaning phase. This approach integrates multiple predictive parameters of weaning success with respiratory muscle ultrasound.
\end{abstract}

\section{Introduction}

Almost half of the patients in the intensive care unit (ICU) require mechanical ventilation (MV), and about $20 \%$ of these patients experience difficult and prolonged weaning from MV [1]. Potential contributors to ventilator-dependence include respiratory dysfunction, cardiovascular dysfunction, neuromyopathy, pharmacological influences, nutritional and psychological factors [2]. Moreover, the limited mobility of critically ill patients and disuse of their inspiratory muscles promotes the early onset of respiratory muscle dysfunction, especially when prolonged mechanical ventilation support is required (i.e., more than 1 week) [3, 4]. In the ICU, respiratory muscle function decreases dramatically, due to alterations in contractile properties that decrease in

\footnotetext{
*Correspondence: paolo.formenti80@gmail.com

${ }^{1}$ SC Anestesia e Rianimazione, Ospedale San Paolo-Polo Universitario,

ASST Santi Paolo e Carlo, Milan, Italy

Full list of author information is available at the end of the article
}

fatigue resistance indexes [5, 6] and atrophy [7]. Although it is likely that the diaphragm is much more sensitive than any other muscle to disuse, intercostal muscles too, along with other major respiratory muscles, are thought to be involved $[8,9]$.

Many tools are available to monitor and evaluate respiratory muscle function $[10,11]$. Among these, the most extensively studied have traditionally been those which evaluate the diaphragm function [12-14]. Current knowledge about the intercostal muscle function primarily has been based on the results of a relatively small number of electromyographic studies relating to chest wall shape and motion [15-17] or to indices of inspiratory effort detected by electromyographic activity [18]. Although providing useful data about muscular activation, this methodology is seldom used in clinical practice and, most importantly, does not provide information about muscle dimensions, shape, and motion. Moreover, it may be prone to low-quality recordings influenced by noise and artifacts, and requires off-line 
analysis performed by a specialist to be interpreted [19]. In contrast, ultrasound may represent a potential modality to integrate these evaluations, assessing muscular activity and structure also in diverse respiratory muscle groups. In particular, ultrasound-guided estimation of muscle dimension (by measurement of muscle thickness) and the ultrasound-guided estimation of weakness (by the assessment of muscle contraction during spontaneous or assisted breathing) has recently been advocated as a simple and reliable method for such a bedside evaluation [20-22]. Similar to the diaphragm, monitoring the function of parasternal intercostal muscles might help to identify and quantify the degree of activation of inspiratory muscles during assisted breathing. Unlike other extra diaphragmatic inspiratory muscles of the neck (such as sternocleidomastoids or scalenes) which are increasingly recruited during voluntary inspiratory tasks [23], the ultrasound window of the parasternal intercostals is almost always accessible in the supine position. Accurate muscle measurement depends not only on operator skills (e.g., measurement location, probe angulation), but also on technical aspects related to ultrasound physics and patient characteristics. While in general ultrasound seems to be a reliable technique, comparing individual patient results must be done with a degree of caution and only after adequate training, as small observer-dependent variations will affect results [24-26]. The aim of this review is to define the role of the intercostal muscles and their ultrasound evaluation during MV. To identify relevant papers, a bibliographic search was conducted accessing the following databases: PubMed, Cochrane Library, Scopus, Web of Science, from inception to the cutoff date of February 29th, 2020. To supplement the search, the reference list of every paper was also manually screened to identify additional potentially eligible studies. The following key-words were used, alone or combined with appropriate Boolean operators, to search the different databases: "intercostal muscle", "extra diaphragmatic muscle", "mechanical ventilation weaning", "respiratory muscle ultrasound", "intercostal muscle ultrasound", "parasternal intercostal muscle ultrasound", "critically ill patients", "critical illness", "intensive care". A similar search was also performed using the PubMed MeSH thesaurus. The final search identified 89 eligible studies, which were subsequently and independently screened and underwent full-text review to identify 21 studies.

\section{Anatomy and physiology of respiratory muscles}

Together with the diaphragm, extra-diaphragmatic inspiratory muscles participate in the generation of the tidal volume. The intercostal muscles are composed of three thin layers of muscle fibers occupying each of the intercostal spaces. The external intercostals extend from the tubercles of the ribs dorsally to the costochondral junctions ventrally, and their fibers are oriented obliquely, downward, and forward, from the rib above to the rib below (Fig. 1, Panel A). Ventrally, between the sternum and the costochondral junctions, the fibers are those of the internal intercostal muscles; these are particularly thick in parasternal region of the rib cage, where they are conventionally called the parasternal intercostals. These anatomical structures are active during the inspiratory phase and interact with the diaphragm and other extra diaphragmatic inspiratory muscles [27]. In contrast, the internal intercostal muscles are directed obliquely, upward, and forward from the superior border of the rib and costal cartilage below to the base of the subcostal border of the rib. The innermost intercostal muscles are the deepest, separated from the internal intercostal muscles by a grouping of nerves and blood vessels known as the neurovascular bundle [15]. These internal intercostals are active during the expiration phase of respiratory cycle (Fig. 1b). The complex interactions among all these fiber groups have been investigated in few physiological humans' studies. The inspiratory drive to the external intercostal muscles was assessed by De Troyer et al. [8], who showed how the third dorsal external intercostal was active earlier in inspiration. Moreover, the degree of activation of the external intercostal muscles during quiet breathing correlated with the magnitude of their mechanical advantage, which diminishes fourfold from the second to the fifth interspace [17]. The same authors, in a complex physiological study, showed how the parasternal intercostals have an inspiratory action on the lung even if with a pressure-generating ability less remarkable as compared to other extra diaphragmatic muscles [16]. Moreover, the study described how the fractional shortening of the parasternal intercostals decreased gradually from the second to the fifth interspace. Nevertheless, it remains unknown whether the neural drive to parasternal intercostal muscles is graded across the interspaces [28]. The spatial distribution of neural drive along rostro-caudal gradient has been demonstrated in a study in which the timing of activation and the discharge performance of single motor units activated during inspiration in the parasternal intercostals of the first to fifth interspaces were measured [29]. During respiratory loading, Sampson et al. [30] showed how the pattern of chest wall deformation was closely related to the activity and coordination of the various inspiratory intercostal muscles, and how the parasternal intercostals do not necessarily represent all inspiratory intercostals. In summary, the movement of the ribs depends on the relative amount of torque around the vertebral articulations acting on the two points of attachment of the muscle to the respective 


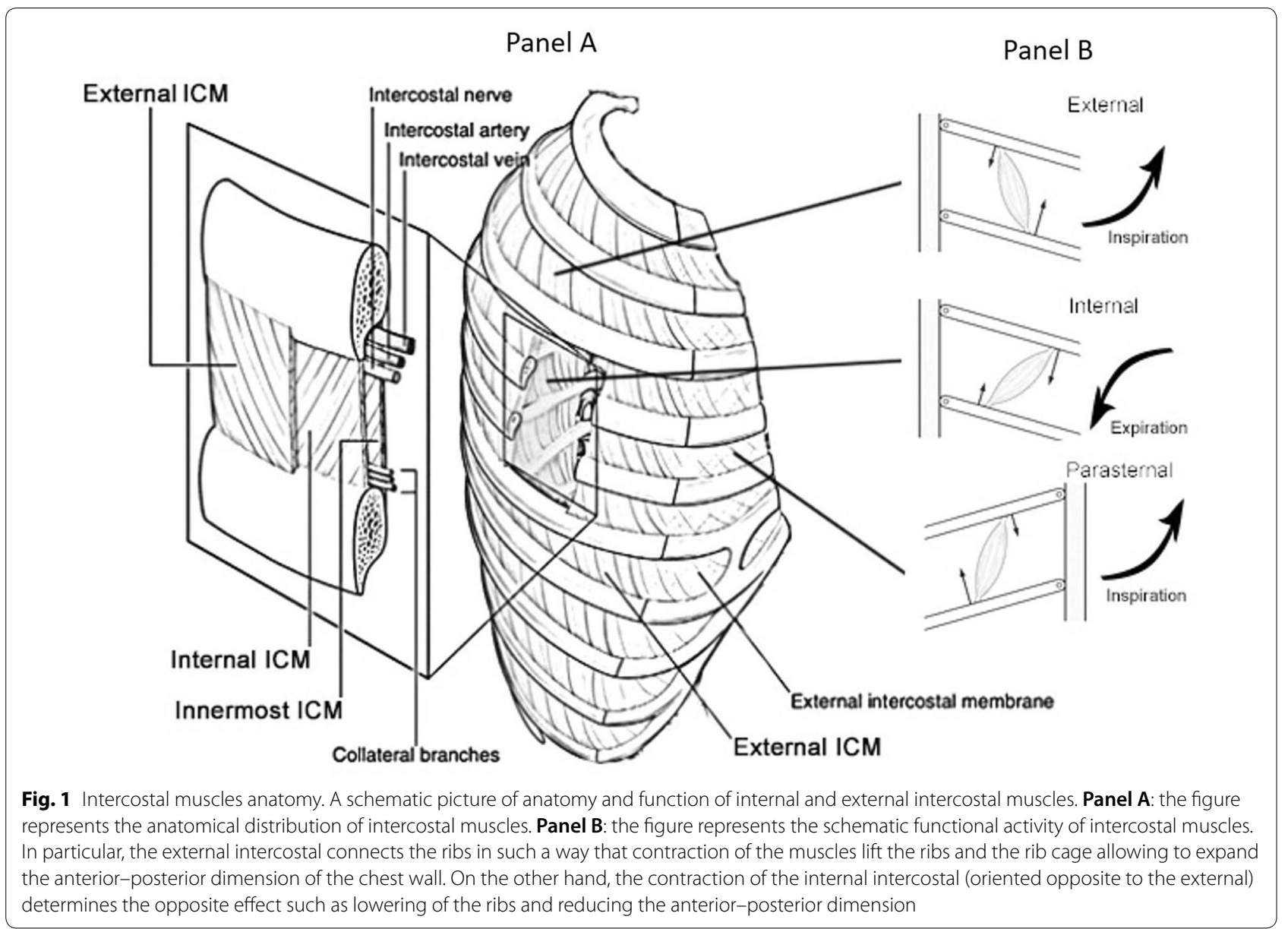

ribs: when external intercostal muscles contract, the torque acting on the lower rib is greater than that acting on the upper rib, with a net effect to raise the ribs. The reverse actually happens for the internal intercostals, which act to lower the ribs to which they attach. Eventually, the parasternal intercostals raise the ribs as their action is transmitted to the sternum (Fig. 1b).

\section{Ultrasound measurements of intercostal muscle}

Ultrasound imaging has been advocated as an interesting imaging modality as it has been shown to be accurate, reproducible and convenient tool for the assessment of linear dimensions, cross-sectional area, thickness, and indices of muscle architecture [31, 32]. In addition, ultrasound has already been used to assess the relationship between muscles and chest wall motion [33, 34]. An animal model demonstrated the ability of ultrasound to accurately estimate the cross-sectional area of the parasternal intercostal muscles, comparing it with the measurement directly performed on the specimen [35]. In healthy volunteers, ultrasound has been used to investigate parasternal intercostal muscles in the easily accessible anterior parasternal region [35, 36]. Conversely, in the lateral and in the posterior part of the intercostal space, the internal and external intercostal muscles often overlap, making the ultrasound detection of both muscle layers impossible. Therefore, the increasing muscle thickness of the external intercostal muscle during inspiration (an estimate of muscle recruitment) cannot be assessed in this location.

Using ultrasound imaging in healthy volunteers, Cala et al. [33] reported that the muscles move ventrally and straighten. Cala et al. [36] measured the inter-rib distance, i.e., the parasternal intercostal muscle thickness. The authors showed a ventral movement during tidal breathing without changes in inter-rib distance and thickness, thereby concluding that the parasternal intercostal muscle moves ventrally and more perpendicular to the ribs. The findings support an intercostal stabilizing function of the parasternal intercostal muscles. Wallbridge [24] postulated that the relationship between intercostal muscle sizes might reflect different roles and recruitment that depends on chest wall site. This hypothesis was based on weak correlation $(r=0.33)$ observed 
between ultrasound thickness of parasternal intercostals and severity of airflow obstruction as measured by spirometric parameters (i.e., FEV1\% predicted) in stable COPD patients. Given the preliminary nature and the weak correlation of the results, this topic deserves to be explored more thoroughly. A recent study by Yoshida et al. [26] used ultrasound to assess whether human parasternal intercostal muscle thickness increased during vigorous breathing efforts (the maximal inspiratory level using an inspiratory resistance loading device). The images obtained showed an increase in muscle thickness only in the anterior portion of the intercostal space during maximal breathing, whereas no significant differences could be detected in the lateral and posterior portions. This remark suggest how the parasternal intercostal portion may be the best region to investigate the intercostal muscle, as in the lateral and posterior part of the intercostal space, internal intercostal muscles and external intercostal muscles overlap, and ultrasound cannot separate both muscle layers. Nevertheless, these observations must be investigated more thoroughly in mechanically ventilated patients. Regarding the reproducibility of ultrasound technique, a high interobserver reproducibility of parasternal intercostal end-expiratory and peakinspiratory thickness has recently been confirmed [37] as previously reported $[35,36]$. Moreover, intra-rater reliability of intercostal muscle thickness depended on site, ranging between 0.87 and 0.97 [24]. Relevant observations are summarized in Table 1.

\section{Ultrasound features and settings}

The parasternal intercostal muscles can be assessed using a 10-15 MHz, linear ultrasonography transducer in M-mode, placed 3-5 cm laterally from the sternum, and oriented transversally in the sagittal plane between the 2nd and the 3rd rib [37, 38]. For instance, while the patient is lying at a $20^{\circ}$ head-up position, the linear array transducer is positioned perpendicular to the anterior thorax surface in the longitudinal scan. Starting in B mode, the pleural line is easily detected as a part of the "bat sign" [39]. Just above the pleural line, the parasternal intercostal muscle can be identified as a three-layered biconcave structure, where two linear hyperechoic membranes running from the anterior and posterior aspects of the adjoining ribs, and a medial portion with muscle echotexture (Fig. 2) [24, 37]. Muscle thickness is measured between the inner and outermost hyperechogenic layer of the muscle fascial borders. Moreover, using the M-mode, the inspiratory contraction of the muscle can be detected, as described for the thickening fraction of the diaphragm [40]. In fact, during the inspiration phase, an increase in muscle thickness can be visualized as the muscle fiber contraction displaces the rib cage cranially and anteriorly, while muscle mass is maintained constant [27]. Parasternal intercostal muscle-thickening fraction (TFic) can be calculated as the ratio of the difference between end-expiratory and end-inspiratory thickness to end-expiratory thickness $(\mathrm{TF}=((\mathrm{TH}$ end inspiration - $\mathrm{TH}$ end expiration)/TH end expiration)) * 100), similarly to the diaphragm thickening fraction (TFdi). Furthermore, indirect qualitative information on muscle composition/structure can be recorded by measuring muscle echogenicity (image-grey-scale) [41]. Muscle echogenicity can define the region of interest for analysis performed using a standard histogram function widely available in many commercial software for image editing [42]. Higher muscle echogenicity (indicating poorer muscle quality) of parasternal intercostal muscle is negatively correlated with COPD severity [24]. However, since echogenicity measurements are influenced by observer-dependent factors more strongly than are other measures, a rigorous ultrasound setting must be selected before acquiring the image to standardize the methods. Some features regarding the probe orientation must also be considered when exploring parasternal muscles. In fact, it has been supposed that the parasternal intercostal muscle image quality progressively reduces as the angle of incidence of ultrasound changes by moving the transducer away from the parasagittal plane. In a pioneering study, Wait et al. [43] showed how the value of the misalignment angle-i.e., the angle at which the transducer no longer produces a readable signal-ranged from $1.6^{\circ}$ to $8.4^{\circ}$ for transducer frequencies between 10 and $1 \mathrm{MHz}$, respectively, during the evaluation of diaphragm movements. Such observations have never been tested for the evaluation of intercostal muscle. Since the ventral and dorsal edges of the parasternal intercostal muscles are not parallel in the majority of the images, the thickness measurements should be performed at the cranio-caudal midpoint between the ribs. There, the difference in the curvature between the two surfaces is least, and the lines flattest.

\section{Parasternal intercostal muscle changes during mechanical ventilation}

Even if only few studies have focused specifically on the intercostal muscles in mechanically ventilated patients, some recent papers investigated the effects of MV on extra diaphragmatic respiratory muscles (Table 1). Starting from animal observations, Capdevilla et al. [44] showed how MV in rabbits produces alterations in contractile properties of the 5th external intercostal muscle, increasing muscle fatigue and promoting atrophy of type II fibers. More specifically, Bernard et al. [45] showed a disruption of myofibrils in both the diaphragm and the 5 th and 6th external intercostal muscles. 


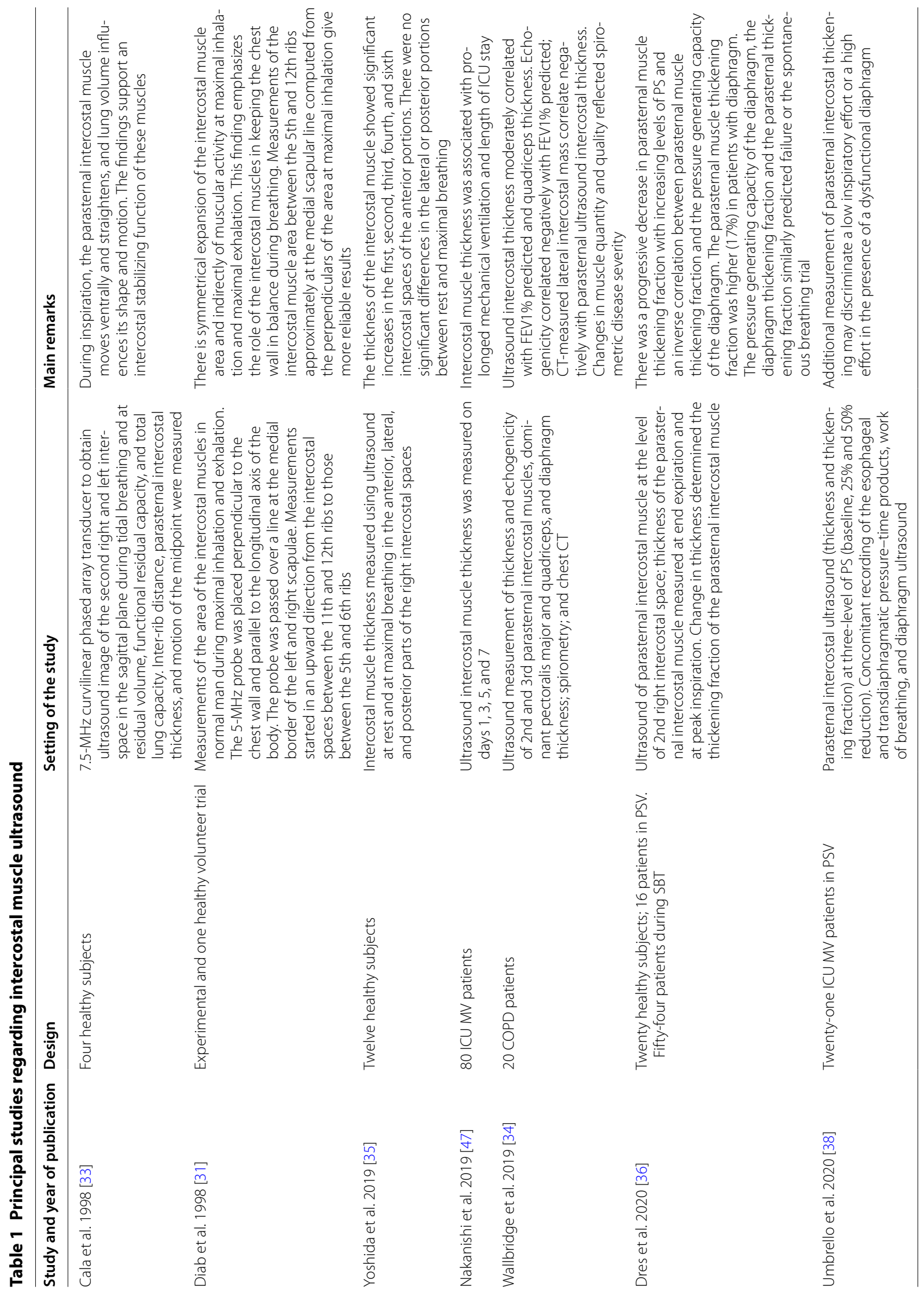


a

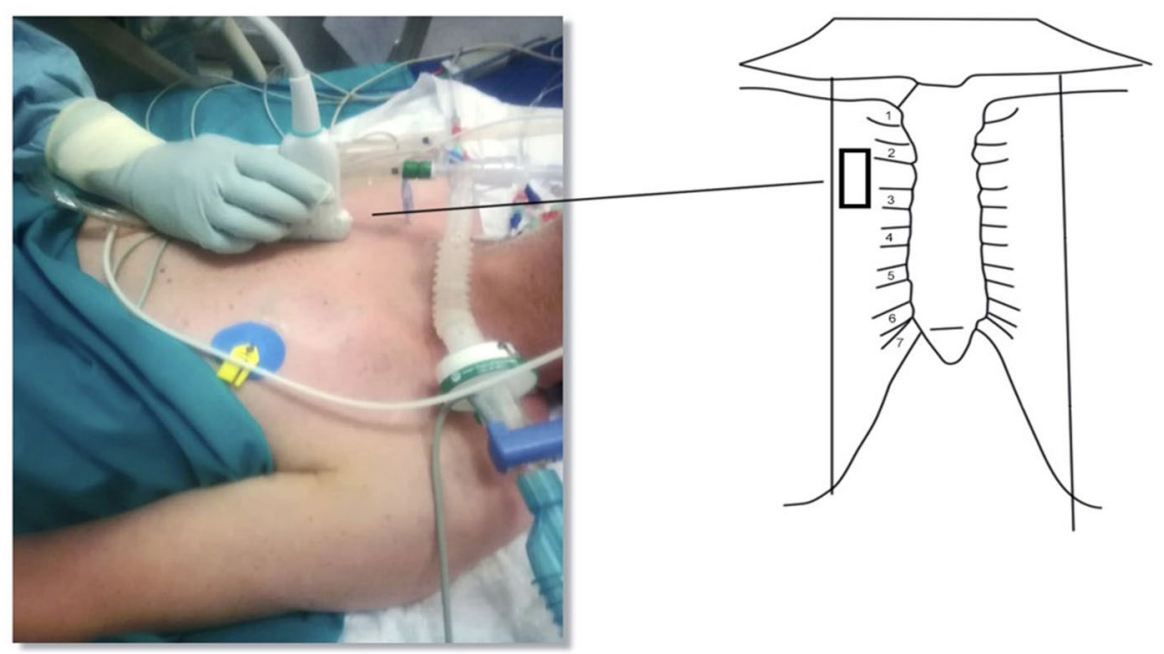

b
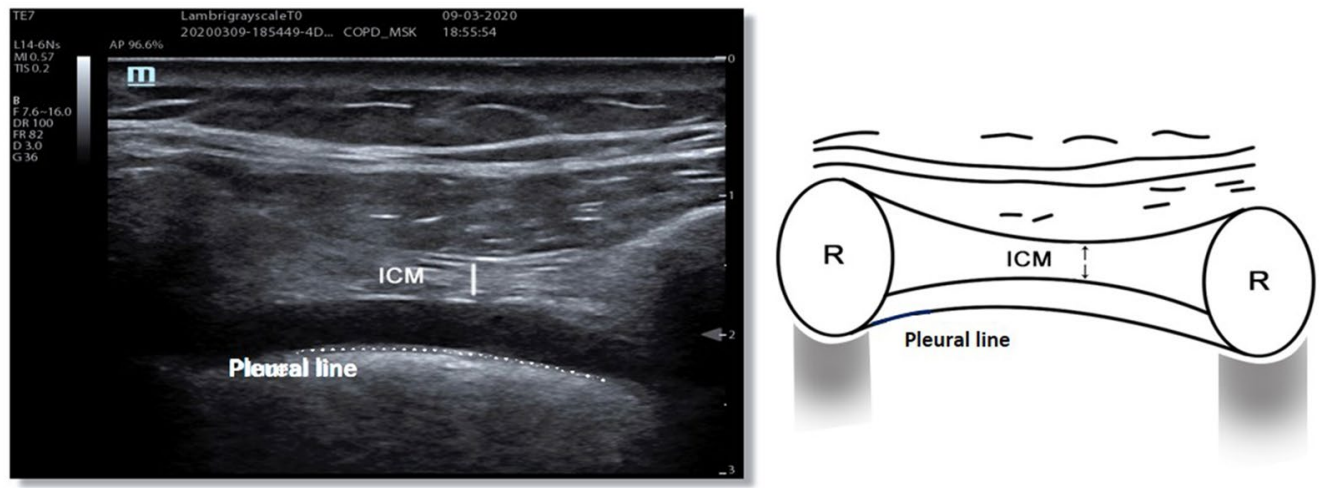

Fig. 2 Intercostal muscle ultrasound. The figure depicts an ultrasound image of parasternal intercostal muscle ultrasound. a The ultrasound probe position at the parasternal space. $\mathbf{b}$ The ultrasound image depicted by ultrasound using a B-mode setting in which the intercostal muscle lies between two ribs (R), cranial to the pleural line, behind the pectoral muscle. The left side is a schematic view of the ultrasound image. ICM intercostal muscle

Direct extrapolation of these scarce results to human subjects may be questioned; however, a time-dependent relationship between the duration of ventilation and the subsequent development of weakness appears likely [37, $46,47]$. In this regard, whereas the effects of MV on the diaphragm have extensively been studied [10, 22], there is little evidence regarding its effects on the performance of other inspiratory muscles and the relative contribution of intercostal muscle dysfunction remains to be determined, especially in ventilator-dependent patients. Nakanishi et al. [48] showed how an excessive inspiratory support causes atrophy of the disused diaphragm and intercostals muscle and that excessive pressure might cause injury to respiratory muscles and structural changes with gradually increasing thickness. Recently, Dres [37] reported that the TFic was significantly associated with failure of a spontaneous breathing trial in mechanically ventilated patients and how this thickening was responsive to the level of ventilator assistance and significantly higher in patients with diaphragm dysfunction. More specifically, a TFic exceeding $8 \%$ identified patients with diaphragm dysfunction, and a value greater than $10 \%$ predicted weaning failure. In support of this observation, Umbrello et al. [38] showed similar results, describing a complex relationship between the diaphragm and parasternal intercostal muscles. The authors showed how diaphragm thickening fraction was higher $(>30 \%)$ and that of parasternal intercostal was lower $(<5 \%)$ in patients without as compared to patients with diaphragm dysfunction. In the presence of diaphragm dysfunction a low TFdi may reflect a low inspiratory effort, or an elevated effort with inspiratory work performed by extra-diaphragmatic muscles associated with low or elevated levels of Tfic, depending on level the mechanical respiratory support 


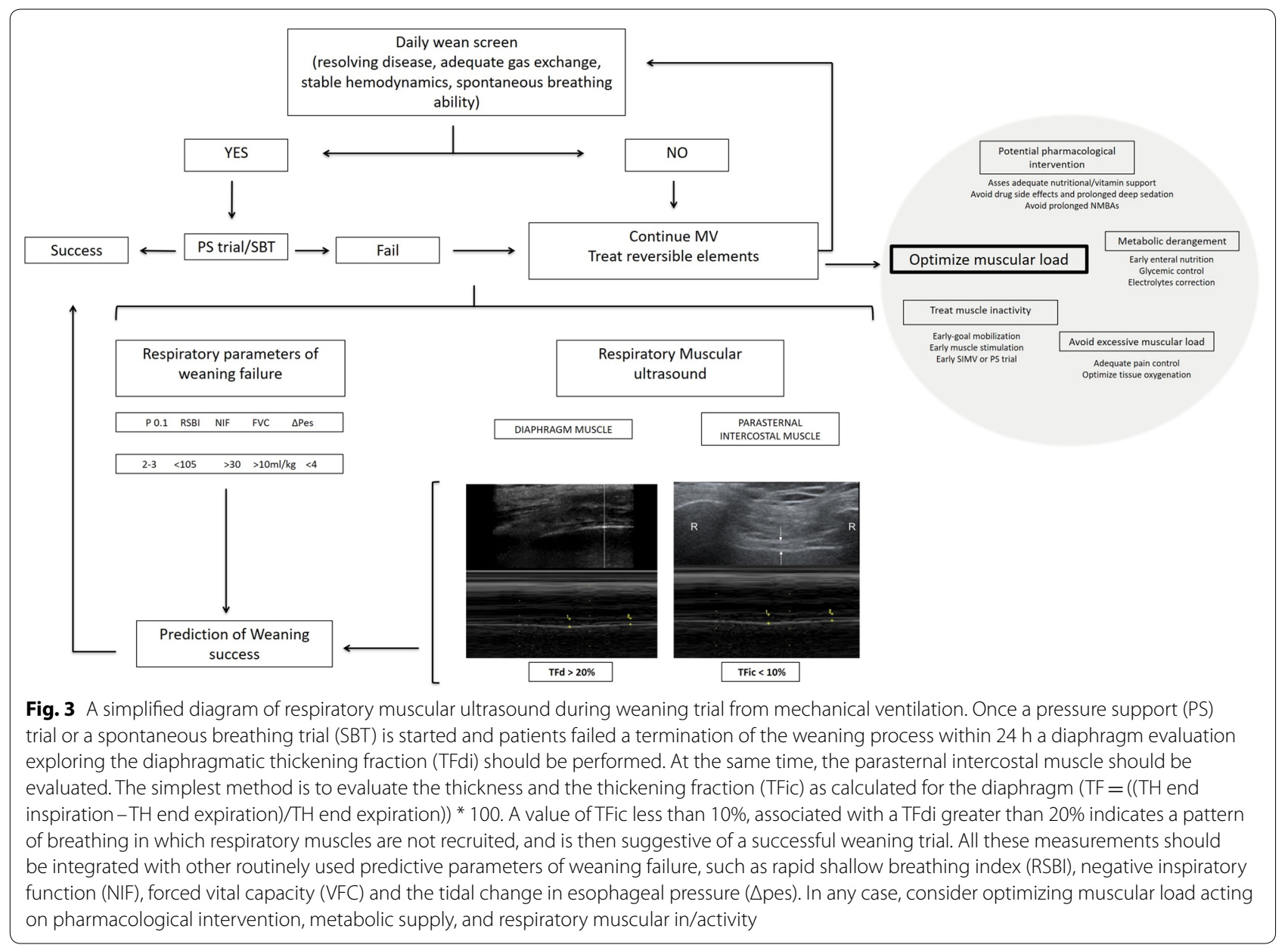

delivered by the ventilator. While we acknowledge that these are recent and preliminary observations, and more importantly given the monocentric and pilot nature of the studies, their consistency and strong physiological rationale led us to propose their use in clinical practice.

\section{Practical approach and potential applications}

Based on the recent consistent findings on this topic [37, 49], we propose a practical flowchart during the weaning trial from $\mathrm{MV}$, in which ultrasound muscular evaluation of the whole respiratory apparatus is taken into account. A similar approach has recently been suggested in an $A B C D$ ultrasound flowchart [49]. Weaning failure has a multifactorial nature, which results from muscular dysfunction, excessive mechanical load, weaning-induced cardiovascular dysfunction, or reduced ability to clear airway secretions. Most physicians simply evaluate the patient's ability to tolerate a spontaneous breathing trial (SBT) without distress to determine likely weaning success $[50,51]$. We propose that once a SBT is started, a diaphragm evaluation exploring the
TFdi should be considered in those patients who failed a termination of the weaning process within $24 \mathrm{~h}$ [1]. At the same time, we propose to evaluate the parasternal intercostal muscles (Fig. 3). This suggestion derives from considering that even when low contractile activity of the diaphragm is identified, two different scenarios may be present: the diaphragm evaluation may be in the normal range because of an overassisted machine setting, or this reduced activity may signify diaphragm dysfunction. In the latter case, the involvement of parasternal intercostals should be considered, as a significant thickening of these muscles may be a sign of muscle recruitment. Thus, a value of TFic less than 10\%, associated with a TFdi greater than $20 \%$ is suggestive of a breath in which the diaphragm is the main inspiratory muscle and the extra diaphragmatic muscle is not "stressed", thereby potentially leading to a successful weaning trial $[37,38]$. Otherwise, it may be possible that, in the presence of diaphragm dysfunction, the intercostal muscles have become more active, which is a negative indicator, potentially predictive of weaning failure. The clinicians 
must consider that these thresholds have not been prospectively validated, and that all these measurements may be integrated with other commonly used predictive parameters of weaning success, such as the rapid shallow breathing index (RSBI), the negative inspiratory pressure (NIP), forced vital capacity (FVC) [52], gas exchange and the tidal changes in esophageal pressure ( $\Delta$ pes) [53]. These, however, are not always investigated, and each is characterized by its own specificity and sensitivity. While we are well aware that it is simplistic to think that a single parameter could predict overall weaning success or failure, we nevertheless think that the strong physiological rationale of parasternal intercostal ultrasound may be of help to the clinician after an adequate training. With the exception of chronic muscular disease [54], the contributions to respiratory failure from respiratory muscles other than the diaphragm, and the possible causative role of prior muscle inactivity, still are poorly understood. No study has specifically investigated the role of intercostal muscles during support by different respiratory devices, such as non-invasive ventilation.

\section{Conclusions}

The available evidence suggests a mechanism by which the parasternal intercostals, and potentially other inspiratory muscles, may contribute to the inspiratory fall in pleural pressure by preventing paradoxical inward displacement of the upper rib cage and decreasing ribcage distortability. Moreover, unphysiological contraction (i.e., thickening) of such muscle groups may be the sign of an excessive recruitment of accessory muscles, such as in the case of underassisted breathing. This may be investigated by using the ultrasound during weaning from MV. Muscle architecture and its complex interrelationship with the muscular and bony rib-cage apparatus require further careful investigation. The intriguing but preliminary nature of the available data highlights the need for more detailed models of intercostal mechanics than presently available to assist our understanding of this complex area of respiratory mechanics during MV.

\begin{abstract}
Abbreviations
ICU: intensive care unit; MV: mechanical ventilation; DD: diaphragmatic dysfunction; TFic: intercostal thickening fraction; TFd: diaphragmatic thickening fraction; SBT: spontaneous breathing trial; NIP: negative inspiratory pressure; RSBI: rapid shallow breathing index; $\Delta$ pes: tidal changes in esophageal pressure; FVC: forced vital capacity.
\end{abstract}

\section{Acknowledgements}

We are grateful to Prof. John J. Marini for his precious help in editing English revision.

\section{Authors' contributions}

PF performed the main literature research and was a major contributor in writing the manuscript and editing figures; $\mathrm{MU}, \mathrm{MD}$ and $\mathrm{DC}$ reviewed the manuscript and the figures for important intellectual content. All authors read and approved the final manuscript.

Funding

No funding for this review.

Availability of data and materials

Not applicable.

Ethics approval and consent to participate

Not applicable.

Consent for publication

Not applicable.

Competing interests

The authors declare that they have no competing interests.

\section{Author details}

${ }^{1}$ SC Anestesia e Rianimazione, Ospedale San Paolo-Polo Universitario, ASST Santi Paolo e Carlo, Milan, Italy. ${ }^{2}$ Sorbonne Université, INSERM, UMRS1158 Neurophysiologie Respiratoire Expérimentale et Clinique, Paris, France. ${ }^{3}$ AP-HP Sorbonne Université. Hôpital Pitié-Salpêtrière, Service de Pneumologie, Médecine Intensive-Réanimation, 75013 Paris, France. ${ }^{4}$ Dipartimento di Scienze della Salute, Università degli Studi di Milano, Milan, Italy. ${ }^{5}$ Centro Ricerca Coordinata di Insufficienza Respiratoria, Università degli Studi di Milano, Milan, Italy.

Received: 18 May 2020 Accepted: 30 August 2020

Published online: 07 September 2020

\section{References}

1. Béduneau G, Pham T, Schortgen F, Piquilloud L, Zogheib E, Jonas M, et al. Epidemiology of weaning outcome according to a new definition The WIND Study. Am J Respir Crit Care Med. 2017;195:772-83.

2. Perren A, Brochard L. Managing the apparent and hidden difficulties of weaning from mechanical ventilation. Intensive Care Med. 2013:39:1885-95.

3. De Jonghe B, Bastuji-Garin S, Sharshar T, Outin H, Brochard L. Does ICU-acquired paresis lengthen weaning from mechanical ventilation? Intensive Care Med. 2004;30:1117-21.

4. Berger D, Bloechlinger S, von Haehling S, Doehner W, Takala J, Z'Graggen WJ, et al. Dysfunction of respiratory muscles in critically ill patients on the intensive care unit. J Cachexia Sarcopenia Muscle. 2016;7:403-12.

5. Booth FW. Effect of limb immobilization on skeletal muscle. J Appl Physiol Respir Environ Exerc Physiol. 1982;52:1113-8.

6. Shanely RA, Coombes JS, Zergeroglu AM, Webb Al, Powers SK. Shortduration mechanical ventilation enhances diaphragmatic fatigue resistance but impairs force production. Chest. 2003;123:195-201.

7. Max SR. Disuse atrophy of skeletal muscle: loss of functional activity of mitochondria. Biochem Biophys Res Commun. 1972;46:1394-8.

8. De Troyer A, Kelly S, Macklem PT, Zin WA. Mechanics of intercostal space and actions of external and internal intercostal muscles. J Clin Invest. 1985;75:850-7.

9. Brichant JF, De Troyer A. On the intercostal muscle compensation for diaphragmatic paralysis in the dog. J Physiol (Lond). 1997;500(Pt 1):245-53.

10. Schepens T, Dres M, Heunks L, Goligher EC. Diaphragm-protective mechanical ventilation. Curr Opin Crit Care. 2019;25:77-85.

11. Laveneziana P, Albuquerque A, Aliverti A, Babb T, Barreiro E, Dres M, et al. ERS statement on respiratory muscle testing at rest and during exercise. Eur Respir J. 2019;53:12.

12. Man WDC, Moxham J, Polkey MI. Magnetic stimulation for the measurement of respiratory and skeletal muscle function. Eur Respir J. 2004;24:846-60.

13. Cattapan SE, Laghi F, Tobin MJ. Can diaphragmatic contractility be assessed by airway twitch pressure in mechanically ventilated patients? Thorax. 2003:58:58-62.

14. Doorduin J, van Hees HWH, van der Hoeven JG, Heunks LMA. Monitoring of the respiratory muscles in the critically ill. Am J Respir Crit Care Med. 2013;187:20-7. 
15. Estenne M, Yernault JC, De Troyer A. Rib cage and diaphragm-abdomen compliance in humans: effects of age and posture. J Appl Physiol. 1985:59:1842-8.

16. De Troyer A, Legrand A, Gevenois PA, Wilson TA. Mechanical advantage of the human parasternal intercostal and triangularis sterni muscles. J Physiol (Lond). 1998;513(Pt 3):915-25.

17. De Troyer A, Sampson MG. Activation of the parasternal intercostals during breathing efforts in human subjects. J Appl Physiol Respir Environ Exerc Physiol. 1982;52:524-9.

18. Ward ME, Eidelman D, Stubbing DG, Bellemare F, Macklem PT. Respiratory sensation and pattern of respiratory muscle activation during diaphragm fatigue. J Appl Physiol. 1988;65:2181-9.

19. Tagliabue G, Ji M, Jagers J, Dean D, Wilde E, Easton P. Limitations of superficial EMG estimate of parasternal intercostal muscle activity. Eur Respir J. 2017;50. https://erj.ersjournals.com/content/50/suppl_61/PA1866.

20. Ueki J, De Bruin PF, Pride NB. In vivo assessment of diaphragm contraction by ultrasound in normal subjects. Thorax. 1995;50:1157-61.

21. Baldwin CE, Paratz JD, Bersten AD. Diaphragm and peripheral muscle thickness on ultrasound: intra-rater reliability and variability of a methodology using non-standard recumbent positions. Respirology. 2011;16:1136-43.

22. Umbrello M, Formenti P. Ultrasonographic Assessment of Diaphragm Function in Critically III Subjects. Respir Care. 2016;61:542-55.

23. Hudson AL, Gandevia SC, Butler JE. The effect of lung volume on the coordinated recruitment of scalene and sternomastoid muscles in humans. J Physiol. 2007;584:261-70.

24. Wallbridge P, Parry SM, Das S, Law C, Hammerschlag G, Irving L, et al. Parasternal intercostal muscle ultrasound in chronic obstructive pulmonary disease correlates with spirometric severity. Scientific Rep. 2018. http:// www.nature.com/articles/s41598-018-33666-7.

25. Dres M, Dubé B-P, Goligher E, Vorona S, Demiri S, Morawiec E, et al. Usefulness of parasternal intercostal muscle ultrasound during weaning from mechanical ventilation. Anesthesiology. 2020;132:1114-25.

26. Yoshida R, Tomita K, Kawamura K, Nozaki T, Setaka Y, Monma M, et al. Measurement of intercostal muscle thickness with ultrasound imaging during maximal breathing. J Phys Ther Sci. 2019;31:340-3.

27. De Troyer A, Kirkwood PA, Wilson TA. Respiratory action of the intercostal muscles. Physiol Rev. 2005;85:717-56.

28. Tokizane T, Kawamata K, Tokizane H. Electromyographic studies on the human respiratory muscles; studies on the activity pattern of neuromuscular units. Jpn J Physiol. 1952;2:232-47.

29. Gandevia SC, McKenzie DK, Plassman BL. Activation of human respiratory muscles during different voluntary manoeuvres. J Physiol (Lond). 1990;428:387-403.

30. Sampson MG, De Troyer A. Role of intercostal muscles in the rib cage distortions produced by inspiratory loads. J Appl Physiol Respir Environ Exerc Physiol. 1982;52:517-23

31. Chi-Fishman G, Hicks JE, Cintas HM, Sonies BC, Gerber LH. Ultrasound imaging distinguishes between normal and weak muscle. Arch Phys Med Rehabil. 2004;85:980-6.

32. Cartwright MS, Kwayisi G, Griffin LP, Sarwal A, Walker FO, Harris JM, et al. Quantitative neuromuscular ultrasound in the intensive care unit. Muscle Nerve. 2013:47:255-9.

33. McCool FD, Conomos P, Benditt JO, Cohn D, Sherman CB, Hoppin FG. Maximal inspiratory pressures and dimensions of the diaphragm. Am J Respir Crit Care Med. 1997;155:1329-34.

34. Sassoon CSH, Caiozzo VJ, Manka A, Sieck GC. Altered diaphragm contractile properties with controlled mechanical ventilation. J Appl Physiol. 2002;92:2585-95

35. Diab KM, Shalabi A, Sevastik JA, Güntner P. A method for morphometric study of the intercostal muscles by high-resolution ultrasound. Eur Spine J. 1998;7:224-8.

36. Cala SJ, Kenyon CM, Lee A, Watkin K, Macklem PT, Rochester DF. Respiratory ultrasonography of human parasternal intercostal muscle in vivo. Ultrasound Med Biol. 1998;24:313-26.

37. Dres M, Dubé B-P, Goligher E, Vorona S, Demiri S, Morawiec E, et al. Usefulness of parasternal intercostal muscle ultrasound during weaning from mechanical ventilation. Anesthesiology. 2020;132(5):1114-25. https://doi. org/10.1097/ALN.0000000000003191.

38. Umbrello M, Formenti P, Lusardi AC, Guanziroli M, Caccioppola A, Coppola $S$, et al. Oesophageal pressure and respiratory muscle ultrasonographic measurements indicate inspiratory effort during pressure support ventilation. Br J Anaesth. 2020;125(1):e148-57. https://doi.org/10.1016/j. bja.2020.02.026.

39. Lichtenstein DA. Lung ultrasound in the critically ill. Ann Intensive Care. 2014;4:1.

40. Umbrello M, Formenti P, Longhi D, Galimberti A, Piva I, Pezzi A, et al. Diaphragm ultrasound as indicator of respiratory effort in critically ill patients undergoing assisted mechanical ventilation: a pilot clinical study. Crit Care. 2015;19:161

41. Sarwal A, Parry SM, Berry MJ, Hsu F-C, Lewis MT, Justus NW, et al. Interobserver reliability of quantitative muscle sonographic analysis in the critically III population. J Ultrasound Med. 2015;34:1191-200.

42. Formenti P, Umbrello M, Coppola S, Froio S, Chiumello D. Clinical review: peripheral muscular ultrasound in the ICU. Ann Intensive Care. 2019;9:57.

43. Wait JL, Nahormek PA, Yost WT, Rochester DP. Diaphragmatic thicknesslung volume relationship in vivo. J Appl Physiol. 1989;67:1560-8.

44. Capdevila X, Lopez S, Bernard N, Rabischong E, Ramonatxo M, Martinazzo $\mathrm{G}$, et al. Effects of controlled mechanical ventilation on respiratory muscle contractile properties in rabbits. Intensive Care Med. 2003;29:103-10.

45. Bernard N, Matecki S, Py G, Lopez S, Mercier J, Capdevila X. Effects of prolonged mechanical ventilation on respiratory muscle ultrastructure and mitochondrial respiration in rabbits. Intensive Care Med. 2003;29:111-8.

46. Powers SK, Wiggs MP, Sollanek KJ, Smuder AJ. Ventilator-induced diaphragm dysfunction: cause and effect. Am J Physiol Regul Integr Comp Physiol. 2013;305:R464-77.

47. Dres M, Demoule A. Beyond ventilator-induced diaphragm dysfunctionnew evidence for critical illness-associated diaphragm weakness. Anesthes. 2019;131:462-3.

48. Nakanishi N, Oto J, Ueno Y, Nakataki E, Itagaki T, Nishimura M. Change in diaphragm and intercostal muscle thickness in mechanically ventilated patients: a prospective observational ultrasonography study. J Intensive Care. 2019;7:56

49. Tuinman PR, Jonkman AH, Dres M, Shi Z-H, Goligher EC, Goffi A, et al. Respiratory muscle ultrasonography: methodology, basic and advanced principles and clinical applications in ICU and ED patients-a narrative review. Intensive Care Med. 2020. https://doi.org/10.1007/s00134-01905892-8.

50. Esteban A, Frutos F, Tobin MJ, Alía I, Solsona JF, Valverdú I, et al. A comparison of four methods of weaning patients from mechanical ventilation. $N$ Engl J Med. 1995;332:345-50.

51. Brochard L, Rauss A, Benito S, Conti G, Mancebo J, Rekik N, et al. Comparison of three methods of gradual withdrawal from ventilatory support during weaning from mechanical ventilation. Am J Respir Crit Care Med. 1994;150:896-903.

52. Tobin MJ. CHEST-ATS guidelines on weaning/extubation ignore scientific principles. Chest. 2017;151:1179-80.

53. Jubran A, Grant BJB, Laghi F, Parthasarathy S, Tobin MJ. Weaning prediction: esophageal pressure monitoring complements readiness testing. Am J Respir Crit Care Med. 2005;171:1252-9.

54. Lo Mauro A, Aliverti A. Physiology of respiratory disturbances in muscular dystrophies. Breathe (Sheff). 2016;12:318-27.

\section{Publisher's Note}

Springer Nature remains neutral with regard to jurisdictional claims in published maps and institutional affiliations. 